\title{
EL CONFLICTO INTERIOR Y SUS SECUELAS. RASTREO A TRAVÉS DEL GLOSSARIUM MEDIAE LATINITATIS CATALONIAE
}

\author{
INNER CONFLICT AND ITS AFTERMATH: \\ A SEARCH THROUGH THE \\ "GLOSSARIUM MEDIAE LATINITATIS CATALONIAE"
}

\begin{abstract}
Resumen: La comparación entre el latín clásico y las lenguas románicas lleva a constatar la evolución del concepto de conflicto. Teniendo presente esa evolución, el estudio de la documentación latina producida en la Cataluña altomedieval y la consulta del material que sirve de base para la elaboración del Glossarium Mediae Latinitatis Cataloniae permiten plantear un problema lexicográfico en torno al sustantivo latino conflictus y comprobar cómo queda cubierto su campo semántico.
\end{abstract}

Palabras clave: Lexicografía latina medieval; Latín y lenguas románicas; Semántica histórica y comparativa.
ANA GÓMEZ RABAL

\begin{abstract}
Comparison between Classical Latin and Romance languages allows us to see the development of the idea of "conflict". Having that progression in mind, this paper focuses on the study of the lexicographical problem which arises from the use of the Latin noun conflictus and its changing semantic field, through the analysis of Latin documents generated in Catalonia in the High Middle Ages, which form the basis of the research for the Glossarium Mediae Latinitatis Cataloniae.
\end{abstract}

Keywords: Medieval Latin Lexicography; Latin and Romance languages; Historical and Comparative Semantics.

\section{SUMARIO}

1. Documentación catalana altomedieval. 2. Conflicto y conflicto interior. 3. Conflicto y decisión. 4. Sin persistencia, substitución.

\section{Documentación CATAlana ALTOMEDIEVAL}

La producción de documentos notariales, como es bien sabido por el público presente, es una rica constante en la Cataluña medieval, y, concreta-

${ }^{1}$ Miembro del equipo de redacción del Glossarium Mediae Latinitatis Cataloniae, cuya elaboración y publicación es fruto de la colaboración entre la Institución Milá y Fontanals del CSIC y el Departamento de Filología Latina de la Universidad de Barcelona. El presente trabajo se ha realizado en el marco del proyecto "Informatización del Glossarium Mediae Latinitatis Cataloniae (5)" (HUM2005-03818/FILO), sufragado por el Ministerio de Educación y Ciencia, ejecutado en la Institución Milá y Fontanals. 
mente, ya en la Cataluña altomedieval desde el siglo IX. La lectura del corpus de documentos latinos catalanes de entre los siglos IX y XII constituye el punto de partida del trabajo lexicográfico que se realiza en el seno del equipo del Glossarium Mediae Latinitatis Cataloniae (GMLC). Esto es, a partir de las ediciones publicadas y alguna vez, si es necesario, a partir de la consulta directa de los manuscritos en los archivos, se elaboran las fichas por entradas, primer eslabón en el proceso de redacción y realización del glosario ${ }^{2}$. Pero, aunque no es mi intención obligarles a llevar a cabo aquí un ejercicio de lexicografía, sin embargo sí querría intentar mostrarles qué se plantea un filólogo, un lexicógrafo que trabaja a partir de una lengua y de unos textos que están "entre dos lenguas", qué se plantea - decíamos- a la hora de acometer el estudio de un término, de un determinado campo léxico o de un determinado campo semántico. Para ello nos va a servir de punto de arranque una voz sonora pero no chirriante, más bien casi obligada, en el contexto de un coloquio que, como el que aquí nos ha reunido, versa sobre las situaciones de conflicto, sean religiosas, sean bélicas, sean de otro tipo - apuntaría yoy de sus vías de solución. Se trata de la voz conflictus, palabra clásica, que no fue recogida en su momento por los redactores del GMLC ${ }^{3}$. Después de tratar sobre ella querría recordarles una serie de términos que se abrieron hueco en el mismo campo semántico que conflictus, con sus propios rasgos de especificidad. Y digo "recordarles" porque son términos que ustedes, historiadores en su inmensa mayoría, habrán encontrado seguramente en sus lecturas.

\section{CONFLICTO Y CONFLICTO INTERIOR}

La definición genérica que se da del substantivo conflictus, - $u s$ (m.) en los diccionarios de latín clásico manejados habitualmente por los latinistas es la de 'choque', 'lucha', 'combate'4. Pero hay que tener en cuenta que el

\footnotetext{
${ }^{2}$ Téngase en cuenta que, en el mes de julio del año 2007, el equipo del GMLC tenía digitalizados, leídos y corregidos 16.255 documentos.

${ }^{3}$ Se puede comprobar en el fascículo 5 (clausa-confrater) del vol. I del Glossarium Mediae Latinitatis Cataloniae, fascículo publicado en 1969. Cf. M. BASSOLS: J. BASTARDAS (dirs.), Glossarium Mediae Latinitatis Cataloniae, voces latinas y romances documentadas en fuentes catalanas del año 800 al 1100 , vol. I (A-D), Barcelona, CSSIC-Universidad de Barcelona, 19601985, y J. BASTARDAS (dir.), Glossarium Mediae Latinitatis Cataloniae, mots llatins i romànics documentats en fonts catalanes de l'any 800 al 1100, fasc. 11 (F), Barcelona, CSIC, 2001 y fasc. 12 (G), Barcelona, CSIC, 2006.

${ }^{4}$ Véase cómo se define en los siguientes diccionarios:
}

GAFFIOT (Dictionnaire illustré latin-francais, Paris, Hachette, 1934): conflictus, -us m. (confligo): 1 [seult à l'abl.]: choc, heurt: CIC, Caec. 43; Nat. 2, 25; Div. 2, 44 || [fig. GELL. 7, 2, $8 \| 2$ lutte, combat: VOP. Car. 10.

[heurt: $m$. choque, encuentro, encontrón, tropiezo, tropezón, tope(tón); golpe; (fig.) conmoción violenta]

Vox (Diccionario ilustrado latino-español, español-latino, Barcelona, Biblograf, $\left.1996^{20}\right)$ : conflictus [abl. $-u$ ] $\mathrm{m}$.: choque, lucha, combate. 
primer sentido recogido de la voz latina conflictus (procedente del verbo confligo, -ere $)^{5}$ hace referencia a un choque o colisión como el que se puede producir entre piedras (Lapidum conflictu atque tritu elici ignem videmus, Cicerón, De natura deorum 2, 25, "vemos que se saca lumbre del choque y roce de las piedras"), al enfrentamiento físico en sí entre personas que luchan, al encuentro violento, al combate o pelea como acción corpórea, exterior, externa. No es el conflictus en principio en la lengua latina clásica un "conflicto" descrito desde el punto de vista figurado o abstracto, no es tampoco un "conflicto" desde el punto de vista moral. El sentido figurado del término aparece por primera vez en Aulo Gelio, gramático del siglo II d. C., que así lo emplea en su obra Noctes Atticae, refiriéndose a lo que podríamos traducir por "asalto del destino" 6 .

Una cuestión muy estudiada y debatida entre los filólogos latinos es el avance de la lengua latina en el camino de la abstracción. En ese proceso, en ese progreso hacia la expresión de la abstracción y, asimismo, en la creación del vocabulario técnico de la filosofía y del conocimiento desempeñó un papel importantísimo Cicerón con su afán de crear una lengua filosófica en latín ${ }^{7}$. Partió de textos griegos y los tradujo (en particular, una parte del Timeo de Platón). Quiso renovar terminológicamente el latín, pero también llevar a

BLÁNQUEZ, Diccionario latino-español español-latino, Barcelona, Sopena, 2002: conflictus, us. (de confligo). m. Gell., Pacat. (sólo en ablat.). Choque, roce. Lapidum conflictu atque tritu elici ignem videmus, Cic. vemos que se saca lumbre del choque y roce de las piedras. \|Vop. Lucha, combate, conflicto. \|(fig.) Gell. Asalto, embate.

Antoni SEVA I LlinaRES (dir.), Diccionari llatí-català, Barcelona, Enciclopèdia Catalana, 1995 [= 1993]: conflictus, us, $m$. xoc, col-lisió, topada: corporum, nubium, dels cossos, dels núvols. ? < confligo.

Cf., asimismo, el Thesaurus Linguae Latinae, Leipzig-München, 1900-, vol. IV 237-238, s. v. conflictus, donde como primera acepción se afirma "generaliter $i$. q. collisio" y como segunda "specialiter $i$. q. certamen, pugna". Nótese, por otra parte, que el término aparece en el diccionario etimológico de A. ERNOUT; A. MEILLET, Dictionnaire étymologique de la langue latine. Histoire des mots, Paris, Klincksieck, $1985^{4}$ ' $\left[=1959^{4}\right]$ (aumentado y corregido por Jacques ANDRÉ), s. v. fligo, verbo traducido como 'battre'.

${ }^{5}$ Vox: confligo -flixi -flictum 3 tr. chocar $\|$ confrontar || intr. entrechocarse, luchar, combatir (cum aliquo c., Tibrar batalla con uno); batirse (armis, arma en mano) \| estar en conflicto (causae inter se confligunt, los partidos están en pugna).

${ }^{6}$ Noctes Atticae 7, 2, 8, donde Crisipo trata del destino, defendiendo su potencia y necesidad, aun cuando quiere demostrar que existe en nosotros la libertad de decisión y de juicio, a propósito de lo cuạl afirma: "Sin vero sunt aspera et inscita et rudia nullisque artium bonarum adminiculis fulta, etiamsi parvo sive nullo fatalis incommodi conflictu urgeantur, sua tamen scaevitate et voluntario impetu in assidua delicta et in errores se ruunt.

${ }^{7}$ Es cierto que antes de Cicerón ya había habido intentos de creación, en latín, de un léxico específico para el discurso filosófico, pero esos intentos no habían sido ni sistemáticos ni lo sưficientemente amplios. No había bastado con las Sententiae - que Cicerón considera de carácter pitagórico- de Apio Claudio el Ciego, con el poema didáctico-filosófico de Ennio Epicharmus, con la también enniana traducción en prosa Euhemerus de la Historia sacra que Evémero escribió hacia el año 403 a. C., ni con el De natura rerum de Lucrecio para formạ un vocabulario filosófico lo suficientemente aposentado y consistente. De todas formas, no caigamos en el error de pensar que esa falta de vocabulario específico significaría o implicaría forzosamente la imposibilidạd de elucubración filosófica en latín; hay algo que me parece obvio, pero que quizás sea necesario apuntar en contra de la categorización de las lenguas: la elucubración como tal es propia del lenguaje humano, independientemente de la mayor o menor riqueza léxica de una lengua en el campo concreto del vocabulario "técniço" filosófico. Otra cosa es que la existencia de un vocabulario específico bien desarrollado, bien apuntalado, pueda ayudar a hacer más fluidas, más directas las discusiones sobre los problemas que el hombre se plantea. Y es ahí donde la labor de Cicerón como traductor cobra su mayor importancia. 
cabo una verdadera renovación conceptual, y todo ello sirviéndose de todos los recursos que le ofrecía la lengua latina, no limitándose a los simples $\operatorname{calcos}^{8}$ y no renunciando nunca a traducir, usando voluntariamente un estilo ligado al de la retórica para buscar la persuasión filosófica sorprendiendo al lector, afectando su sensibilidad y su imaginación ${ }^{9}$. Con conflictus se podría ejemplificar una vez más el avance de la lengua latina en el camino de la abstracción, un progreso en el que, sin embargo, el peso de lo concreto mantuvo su larga influencia durante tiempo.

En el caso de conflictus en la lengua latina clásica, de la Antigüedad, no se constata la intervención directa de un autor, de un traductor, de un Cicerón que diera intencionadamente un vuelco al significado del término. Pero demos un paso más y fijemos nuestra atención por un momento en el latín que se denomina en Filología Clásica "latín cristiano", esto es, el que abarca desde los inicios de la literatura cristiana, es decir, la época de Tertuliano (principios del siglo III) y de los Padres de la Iglesia, hasta el final de la época merovingia, esto es, a mediados del s. VIII ${ }^{10}$. A lo largo de estos cinco siglos, conflictus, sin perder su significado original, se emplea también con acepciones predominantemente figuradas o abstractas: 'combate' o

${ }^{8}$ Ejemplo de calco: concentio (Timeo 37 A, posiblemente acuñación ciceroniana; procede de concino 'cantar o tocar a la vez' y, en sentido figurado, 'estar de acuerdo', 'concordar', 'estar en armonía') por harmonia. Vid. Ana GóMEZ RABAL, La formación del lengúaje filosófico'latino. Cicerón y Calcidio traductores del "Timeo" de Platón, en Jacqueline HAMESSE (ed.), Roma magistra mundi. Itineraria culturae medievalis. Mélanges offerts au Père L.E. Boyle à l'occasion de son $75 e$ anniversaire, Louvain-la-Neuve, Fédération Internationale des Instituts d'Etudes Médiévales, 1998 (Textes et Etudes du Moyen Âge, 10/3), pp. 73-82, concretamente pp. 77-80.

${ }^{9}$ Cicerón, aun viendo la posible antinomia entre el estilo filosófico y el oratorio, no renuncia a "conmover" y postula, como Aristóteles y Platón, que el discurso fílosófico no sólo debe ser riguroso, sino bello, e intenta armonizar su propio gusto estilístico con la refilexión filosófica. En consonancia con esa visión de la filosofía, rechaza el empleo de una terminología estrictamente técnica. Sobre esta idea, vid. Noemi LAMBARDI, Il "Timaeus" ciceroniano, arte e tecnica del "uertere", Firenze, Le Monnier, 1982 (Quaderni di Filologia Latina, 2), especialmente las pp. $18-21$.

${ }^{10}$ Para fijar los límites de lo que debe entenderse por latín cristiano y, por ende, para agrupar a los autores que se deben incluir en un diccionario "de latín cristiano" Albert BLAISE inicia el prefaçio a su Dictionnaire latin-français des auteurs chrétiens, Turnhout, Brepols, 1954 (revisado especialmente por lo que al vocabulario teológico se refiere por Henri CHIRAT), con las siguientes palabras: "Les auteurs qui font l'objet de ce dictionnaire s'échelonnent sur une vaste période: depuis les débuts de la littérature chrétienne, c'est à dire l'époque de Tertullien, de la Passio Perpetuae, jusqu'à la fin de la période mérovingienne. A vral dire, cette littérature se perpétue jusqu'à la Renaissance, même au-delà, et il est bien artificiel d'établir à un moment quelconque une sorte de coupure dans ce long développement: la création de mots nouveaux, de sens nouveaux ne s'est jamais arrêtée. Sans doute, à l'époque carolingienne, le latin est-il devenu scolaire et inintelligible à la foule, tandis qu'aux $4^{e}, 5^{\mathrm{E}}$ siecles le latin des Pères, tout en étant une langue de culture, n'en restait pas moins compris des auditeurs de sermons. Mais comment fixer une date? Elle serait d'ailleurs différente selon les régions, les milieux. Quoi qu'il en soir, les ravages apportés par les invasions germaniques ont été moins terribles, moins mortels pourrait-on dire, que ceux qui ont été provoqués par la conquête ou les incursions sarrazines, sans parler de la corruption interne et de l'ignorance qui sévit a la fin de la période mérovingienne. Il est donc permis de parler d'une renaissance carolingienne, si éphémère fût-elle, et de fixer à partir de là le début du latin médiéval, tandis que la période mérovingienne constitue comme un long appendice à l'âge patristique proprement dit." 
'batalla' en sentido literal, pero, asimismo, 'lucha' en sentido literal y figurado y sobre todo 'debate' y 'discusión'11.

Demos ahora otro salto: se ve de forma clara que las acepciones figuradas y abstractas de conflictus no sólo se conservan sino que adquieren una mayor relevancia que el sentido literal, físico, si nos atenemos a las definiciones que se dan de él en los diccionarios de las lenguas románicas, en los que se define conflicto $^{12}$, conflicte $^{13}$, conflit $^{14}$, conflitto $^{15}$. En todos ellos el

${ }^{11}$ Véase al respecto:

BLAISE, Dictionnaire latin-francais des auteurs chrétiens: conflictus, -us, $\mathrm{m}$. (cl.) -1. combat, bataille (VOP.): HeGES. 1, 30, 7-\| (fig.) lutte: LEO-M. Serm. 21 (20), $1 \frac{\mathbf{2}}{2}$.

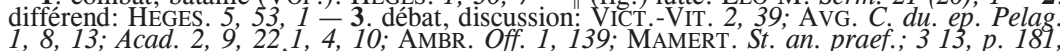
8 .

${ }^{12}$ Véanse al respecto:

Diccionario de la lengua española, Real Academia Española, 200122 [DRAE] conflicto (Del lat. conflictus). $m$. Combate, lucha pelea. U. t. en sent. fig. I' 2 . Enfrentamiento armado. 3. Apuro, situación desgraciada y de difícil salida. || 4. Problema, cuestión, materia de discusión. Al 5. Psicol. Coexistencia de tendencias contradictorias en el individuo, capaces de generar angustia y trastornos neuróticos. $\| 5$. desus. Momento en que la batalla es más dura y violenta. $\|$ colectivo. $m$. En las relaciones laborales, el que enfrenta a representantes de los trabajadores y a los empresarios".

María Moliner, Diccionario de uso del español, Madrid, Gredos, $1997^{20}$ [=1966]: conflicto. 1 Momento más violento de un *combate. • Momento en que el combate está indeciso. 2 («Causar, Mover, Ocasionar, Promover, Suscitar un c. de» o «entre»). Choque, o situación permanente de *oposición, *desacuerdo o *lucha entre personas o cosas: 'Un incidente de fronteras provocó un conflicto entre los dos países. Conflicto de jurisdicciones [de pasiones, de intereses],. (V.: «CHOQUE, COLISIÓN, CUESTIÓN, *DESACUERDO, DIFERENCIA, DISCREPANCIA *DISCUSIÓN, DISGUSTO, ENCUENTRO, PUGNA. • *CONTRARIO, *LUCHA. *OPOSICIÓN.») 3 («Estar», etc., «en, Tener un»). Situación en que no se puede hacer lo que es necesario hacer o en que no se sabe qué hacer: 'Se encontró en un conflicto porque no podía pagar la letra. Tiene un conflicto porque la han invitado a la vez a dos fiestas'. (V. «*APURO»).

[La cursiva se emplea en el diccionario de María Moliner para "texto de los artículos de palabras no usuales"]

Julio CASARES, Diccionario ideológico de la lengua española, Barcelona, Gustavo Gili, $1997^{2}$ : *conflicto m. Lo más recio de un *combate. Momento en que aparece incierto el resultado del $*$ combate. $\|$ fig. Lucha interior, *desasosiego. \| fig. Apuro, *dificultad, *peligro.

[Cuando se busca en la lista de sinónimos y conceptos afines, la parte II "Parte analógica", frente a la III ("Parte alfabética"), s. v. conflicto se remite directamente a dificultad]

Consúltense, además, J. COROMINAS, Diccionario crítico etimológico de la lengua castellana, vols. I-IV, Madrid, Gredos, 1954-1957, concretamente el vol. I, s. v. afligir, y J. COROMINAS; J.A. PASCUAL, Diccionario crítico etimológico castellano e hispánico, vols. I-VI, Madrid, Gredos, 1980-1991, en concreto el vol. I, $s$. v. afligir. En ambas obras, en la categoría de los derivados del verbo latino fligere, 'golpear', se trata del verbo confligere, 'chocar', y, como derivado de este verbo, del término latino conflictus y del romance conflicto.

${ }^{13}$ Véanse al respecto:

Pompeu FABRA, Diccionari general de la llengua catalana, Barcelona, Edhasa $1984^{19}$ (revisado y ampliado por Josep MIRACLE): conflicte $m$. Xoc contesa hostil; esta d'oposició; contesa prolongada. Durant el conflicte de les dues hosts. El conflicte greco-turc. Promoure, resoldre, un conflicte. I Un conflicte d'idees, d'interessos, de passions. I Abs. Trobar-se en un conflicte, indecís entre dos cossos polítics, judicials, etc., que es disputen un dret, una atribució, etc. Un conflicte entre la jurisdicció civil i l'eclesiàstica.

Diccionari de la llengua catalana, Barcelona, Enciclopèdia Catalana, 1994 [= $1993^{3}$ ]: conflicte m. 11 Xoc, contesa, lluita. El conflicte amb la cavalleria enemiga. 2 p ext Contesa prolongada, estat d'oposició. El conflicte àrabo-israelià. 3 fig. Un conflicte d'idees, d'interessos, de passions. Ha entrat en conflicte amb el batlle. 4 PSIC. Situació donada per una discordança entre les tendències o els interessos d'algú i les imposicions externes. 2 fig. Problema, cuita, compromís. Em trobo en un conflicte. 
significado original de 'choque' entendido físicamente sigue apareciendo, pero en todos ellos se desarrolla mucho más el sentido no literal. Paul Robert, concretamente por lo que a la lengua francesa se refiere, califica de significado "antiguo" (vieux, en el diccionario) el de "lutte, combat" ("lucha, combate'), que recoge de La Fontaine ("Le pigeon profita du conflit des voleurs"). Y, frente a ello, define el conflit del francés moderno como "rencontre d'éléments, de sentiments contraires, qui s'opposent" (“choque de

Diccionari de la llengua catalana, Institut d'Estudis Catalans, $2007^{2}$ [extraído de la página web del IEC el 29/IX/2007, consulta libre]: conflicte $11 \mathrm{~m}$. [LC] Contesa, lluita. Durant el conflicte de les dues hosts. $12 \mathrm{~m}$. [LC] Contesa prolongada. El conflicte grecoturc. $13 \mathrm{~m}$. [LC] [SO] Estat d'oposició. Promoure, resoldre, un conflicte. $14 \mathrm{~m}$. [LC] [PS] [PE] per ext. Un conflicte d'idees, d'interessos, de passions. 15 [LC] [PS] [PE] trobar-se en un conflicte Estar indecís entre interessos, desitjos, etc., incompatibles. $16 \mathrm{~m}$. [LC] [SO] [AD] Contesa entre dos organismes polítics, judicials, etc., que es disputen un dret, una atribucio. Un conflicte entre la jurisdicció civil i l'eclesiàstica. ' 1 7 7 [ECT] conflicte col lectiu Situació d'enfrontament entre sindicats i patronal, o entre treballadors 1 empresari, per desacord en qüestions salarials i laborals. 18 [SO] conflicte de classes Conflicte entre classes socials quan una es considera superior $i$ domina les altres $i$ les explota. 19 [SO] conflicte social Desequilibri temporal en la cohesió d'un sistema social, causat per elements dissidents que pretenen de restablir un nou equilibri, fonamentat en unes bases noves. 110 [SO], [ $\mathrm{FL}$ ] conflicte lingüístic Conflicte entre grups en què les diferències idiomàtiques esdevenen el símbo fonamental de l'antagonisme. $2 \mathrm{~m}$. [PS], [PE] En psic., situació donada per una discordança entre les tendències o els interessos d'algú i les imposicions externes.

Antoni M. Alcover; Francesc de B. Moll, Diccionari català-valencià-balear, Palma de Mallorca, Moll, 1978-1979: CONFLICTE $m$. . cast. conflicto. \| 1. Topada hostil, lluita; estat d'oposició. Isqueren a camí al hostol..., e haüt conflicte entre ells..., foren desbaratats, doc. a. 1435 (Ardits, I, 326). Allà on fon lo major conflicte de la batalla, Tirant, c. 328. Al millor dia hi haurà conflicte, Vilanova Obres, IV, 58. \| 2. Cas de dificultat greu, enutjós de
resoldre. Per tants conflictes qui són en tu..., aconsolar te vull un tant, Spill 15000 . Un caualler... trobà lo gran conflicte com son senyor lo rey era mort, Tomic Hist. 41. [...] ETIM.: pres del lilatí conflictum, 'topada', 'lluita'.

$C f$, asimismo, J. COROMINES, Diccionari etimològic $i$ complementari de la llengua catalana, vols. I-IX, Barcelona, Curial, 1980-1991, vol. X (suplement-índex), Barcelona, Curial, 2001, concretamente el vol. I 65b, s. v. afligir, donde el autor, frente al verbo confligir, "rar en català", atestigua las primeras apariciones de conflicte "ja en Joanot Martorell: «e dona batalla als moros, hon hagué molt gran conflicte, que y morí molta gent de una part e d'altra» [T. lo B. I, 22], i en Jaume Roig [«per tants conflictes / que són en tu, Paconsolar / te vull un tant», Spill, v. 15.000]”.

${ }^{14}$ Véase al respecto:

Le Nouveau Petit Robert, Paris, Dictionnaires Le Robert, 1993 (texto retocado y ampliado bajo la dirección de Josette REY-DEBOVE y Alain REY): CONFLIT [...] n.m.- in XII ${ }^{\mathrm{e}}$; bas lat. conflictus «choc» 1 . vX Lutte, combat. «Le pigeon profita du conflit des voleurs» (La Font.). 2. MOD. Rencontre d'éléments, de setiments contraires, qui s'opposent. $\Rightarrow$ antagonisme, conflagration, discorde, lutte, opposition, tiraillement. Conflit d'intérêts, de passions. Conflit de générations, entre parents et enfants, adultes et jeunes. Conflit social. Entrer en conflit avec qqn. «elle avait jusqu'alors évité les conflits ouverts» (Mauriac). $\Rightarrow$ clash. Conflit mineur. $\Rightarrow$ FAM. guéguerre. PSYCHOL. Action simultanée de motivations incompatibles; son résultat. Conflit affectif. 3. Contestation entre deux puissances qui se disputent un droit. $\Rightarrow$ crise. Conflits internationaux. Arbitrage d'un conflit. Conflit armé. $\Rightarrow$ guerre. En cas de conflit. 4. DR. Rencontre de plusieurs lois, textes, principes qui se contredisent et, de ce fait, ne peuvent être appliqués. Conflit de lois. - Conflit de juridiction, entre deux tribunaux pour juger une affaire. Conflit d'attribution. Le tribunal des conflits, chargé d'attribuer la compétence administrative ou judiciaire à une affaire $(\Rightarrow$ litige $)$. CONTR. Accord, paix.

${ }^{15}$ Véase al respecto:

Dizionario Garzanti della lingua italiana, realizzato dalla redazione lessicografica Garzanti diretta da Giorgio Cusatelli, Milano, Garzanti Editore, 1965: conflitto, s. m. 1. combattimento, scontro di eserciti: il - conflitto anglo-boero 2. (fig.) urto, contrasto violento: - conflitto di idee, di interessi, di sentimenti / - psichico, contrasto tra gli impulsi dell'inconscio e la sfera della concienza, oppure tra due gruppi di istinti. // Dal lat. conflictus - us 'urto, lotta, battaglia', deriv. di conflictus, p. p. di confligere 'cozzare insieme, combattere'. 
elementos, de sentimientos contrarios, que se oponen"). En el diccionario de la Real Academia de la Lengua Española o en el del Uso del español, de María Moliner, se recoge específicamente la acepción de conflicto como momento de mayor violencia o fragor en el combate, pero calificando tal acepción de "desusual"; en ambos diccionarios se da relevancia al sentido figurado y abstracto que implica un "conflicto de jurisdicciones, de pasiones, de intereses" resultado ya sea de un choque ya sea de una situación permanente de oposición, desacuerdo o lucha entre personas (un conflicto entre generaciones sería un ejemplo) o cosas. Y, además, en ambos diccionarios se hace hincapié en una acepción más de conflicto: el que genera en uno mismo una situación en la que no "no se puede hacer lo que es necesario hacer o en que no se sabe qué hacer" (María Moliner), una situación de "apuro, [...] desgraciada y de difícil salida" (DRAE), una situación de desasogiego, de dificultad o que incluso puede entrañar peligro para uno mismo (Julio Casares). Estar en un conflicto es estar en un aprieto, estar en un brete, con la lucha interior que eso implica.

Conflicte en catalán, definido también en todos los diccionarios en primer lugar como "xoc, contesa", choque y contienda física, pasa de lo concreto a la extensión de lo que dura ("conflicte àrabo-israelià" en el diccionario del IEC de 1994). Pero se destaca también el sentido figurado que ya hemos descrito: conflicto en un sentido personal, en cuanto afecta a la persona en su debate interior, sus problemas, sus cuitas.

Pero, teniendo en cuenta la evolución señalada, la ampliación del concepto de conflicto, ¿qué podemos decir con respecto al latín medieval? ¿Consolidó el latín medieval algo que el latín clásico, como ya hemos indicado, sólo apuntaba pero que el latín cristiano asentó y amplió? ¿Anunció el latín medieval lo que de forma diáfana se muestra ya en las lenguas románicas, como también hemos visto? Y, ciñéndonos concretamente a lo que al latín medieval del dominio del catalán respecta, ¿qué nos permiten constatar los textos conservados? Recalquemos al respecto que, como he empezado diciendo al iniciar mi intervención, es vastísima la producción de textos en la Cataluña altomedieval, de textos documentales, esto es, actas notariales que transcriben donaciones, dotaciones, ventas, testamentos, juramentos de fidelidad y litigios. Junto a ello y frente a ello, existen unos pocos textos literarios, textos literarios en el sentido amplio del término, el que incluye las bellas letras pero también las obras de carácter histórico, filosófico o científico. En todo caso, unos textos y otros se erigen en el testimonio escrito de la lengua latina medieval, expresión - como es de esperar- del lenguaje jurídico, eclesiástico, institucional y curial de la Alta Edad Media, en la que aparecen importantes innovaciones léxicas junto con claros indicios fonéticos y morfosintácticos de la incipiente lengua romance, el catalán.

Los diccionarios de latín medieval no dejan lugar a dudas: aunque el término se sigue empleando en el sentido literal de 'choque' y también en el de 'batalla', se utiliza sobre todo como abstracto con los sentidos de 
'discusión' e incluso 'argumento' y se especializa como 'duelo judicial'16; pero además se añade un nuevo significado, tardío, recogido en el siglo XIII: el de 'derrota'"

\section{CONFLICTO Y DECISIÓN}

En el conjunto de textos latinos altomedievales catalanes que tenemos a nuestra disposición en la base de datos que está elaborando el equipo del GMLC, esto es, en los más de 16.000 documentos digitalizados y leídos - como hemos dicho más arriba-, la palabra conflictus aparece una sola vez. La aparición se da, además, en un documento que podríamos calificar de tardío si nos atenemos a lo que marcan los límites cronológicos del Glossarium, los años 900 y 1100 según rezaba el subtítulo del vol. I (A-D), cuyos fascículos se publicaron entre el año 1960 y $1985 .{ }^{18}$

Debemos recordar, que, como también hemos indicado antes, no hay una entrada conflictus en nuestro glosario. Se pueden suponer tres razones para intentar justificar la no aparición:

1.- Quizás porque, simplemente, los redactores del Glossarium en los años de preparación del fascículo 5 (que apareció, recordemos, en 1969) ${ }^{19}$ no encontraron ningún ejemplo de uso de conflictus y, en particular, no tuvieron

\footnotetext{
${ }^{16}$ Acompañado en este caso muy a menudo por el adjetivo legalis; $c f$. DU CANGE, Glossarium Mediae et Infimae Latinitatis, conditum a Carolo du Fresne, domino Du Cange, ed. nova a L. Favre, vols. I-X, Niort, 1883-1887, s. v. conflictus: "CONFLICTUS LEGALIS, Duellum ex lege statutum, in Charta Roberti reg. Franc. ex parvo Reg. S. Germ. Prat."

${ }^{17}$ Véanse al respecto:
}

Du CANGE: Conflictus, Substant. Exercitus dissipatio, fuga, Gall. Déroute, apud eumd Bartholom Scribam ann 1242 Praedictos inimicos volentes intrare civitatem posult in Conflictu, apud Anonymum in Chronico Veronensi ad ann. 1317. Dominus Canis fuit positus in Conflictum cum ejus militia et comitiva, multis ex suis interfectis et captis, ibid. ad ann. 1320.

A. BLAISE, Dictionnaire latin-français des auteurs du Moyen-Âge. Lexicon Latinitatis Medii Aevi, Turnholti, Brepols, 1998 [ $=1975]$ (Corpus Christianorum, Continuatio Medievalis): I conflictus, mis en dẻroute. II conflictus, - us (cl. et lat. chr.) - 1. duel judiciaire: c. legalis $-\mathbf{2}$. déroute.

J.F. NIERMEYER, Mediae Latinitatis Lexicon Minus, Leiden-New York-Köln, Brill, 1993: conflictus (decl. iv): 1. *bataille - battle. 2. *discussion - argument. 3. duel judiciaire - judicial combat. Si ad conflictum causae descenderint. Lex Burgund., tit. 80. 4. déroute, défaite - defeat. S. xiii, Ital.

J.H BAXTER; ChARLES JOHnSON, Medieval Latin Word-List from British and Irish Sources, London, Oxford University Press - Geoffrey Cumberlege, 1950 [ $\left.=1934^{1}\right]$ : conflictus (second decl.), fight 6c.; (fourth decl.), dispute 8c.

${ }^{18}$ Aunque se fijaron esos límites cronológicos, eso no fue óbice para que, en los artículos de los fascículos del primer volumen, se incluyeran ejemplos del siglo XII. En relación con ello hay que subrayar que para el proceso de digitalización se ha fijado un límite más amplio: el año 1200. A pesar de que los documentos del siglo XII suelen dar indicios ya de que la lengua latina hă cambiado: los escribas o los notarios tienen una mayor conciencia de que hay que "escribir bien" por lo que en los textos del siglo XII puede que queden más borradas las huellas de la lengua románica y que su latín se convierta en más artificiọso y "correcto" que el de los siglos anteriores; eso quizás es producto del llamado "Renacimiento del siglo XII", pero también de una circunstancia política de suma importancia como fue el matrimonio de Ramón Berenguer IV en 1137 con Petronila de Aragón.

${ }^{19} \mathrm{Cf}$. supra, apartado 1 , nota 3 . 
acceso al documento en cuestión. Apuntemos, en relación con ello, que la digitalización de documentos que ha podido llevar a cabo el equipo del GMLC desde el año 2000 —desde que dispone de los instrumentos informáticos necesarios para aprovechar el potencial que supone la publicación del enorme número de ediciones de cartularios medievales que en las dos últimas décadas han proliferado en Cataluña- permite analizar muchos más textos y crear concordancias o índices alfabéticos de las palabras de los textos dentro de un contexto significativo, lo cual sirve para completar el trabajo realizado en el proceso de creación del fichero original del glosario. Sin embargo, por lo que se ha podido comprobar en la redacción del fascículo de la letra G (2006), la cantidad no hace sino corroborar, por lo general, la calidad del fichero inicial: se encuentran más ejemplos (algunos más antiguos), se completan significados (y a veces se modifican), pero, en el caso de la $\mathrm{G}$, no hubo que añadir ni una sola nueva voz con respecto al vaciado realizado a partir del fichero manual ${ }^{20}$.

2.- Quizás porque localizaron el documento, pero lo consideraron demasiado tardío. No obstante hay que tener presente que, aunque se fijaron los límites cronológicos de los años 900 y 1100, eso no fue óbice para que en los fascículos del primer volumen se incluyeran ejemplos del siglo XII ${ }^{21}$.

3.- Quizás porque, a pesar de haber localizado el documento, consideraron excesivamente poco frecuente la aparición del término como para incluirlo en la publicación.

Vamos a hacer un poco de elucubración lexicográfica, poniéndonos en la piel de los redactores de aquellos momentos -imaginándonos que

\footnotetext{
${ }^{20}$ Conviene tener presente, de todas formas, la necesaria coexistencia de los dos ficheros existentes, el manual y el resultante de la digitalización de documentos. El proyecto de realización del GMLC, siguiendo el camino abierto en la década de 1990, se vertebra actualmente en dos líneas de trabajo que cabe definir como de redacción, la primera, y de digitalización, la segunda. En esta segunda linea se planteó inicialmente la informatización del fichero manual, pero pronto se dirigió tal línea hacia un nuevo fin y, así pues, la informatización de la base documental se dịige en la actualidad, principalmente, a la incorporación de fuentes de edición reciente, teniendo siempre en cuenta criterios de calidad en la selección de las ediciones. Se pretende crear așí, en un soporte magnético, un fichero rico, inédito, abierto a posibles variaciones y ampliaciones (exigidas, sin duda, por el vaciado de nuevas publicaciones documentales), coherente, inteligible y de fácil manejo. Esta labor de digitalización permite, además, la creación y utilización de concordancias, o índices alfabéticos de las palabras de los textos dentro de un contexto significativo, que confirman, completan y amplían la información recogida en la fichas a partir de las que, en principio, trabaja el equipo de redacción del GMLC. El trabajo de digitalización y corrección de documentos a partir de ediciones y de transcripciones alcanza en la actualidad - como se ha dicho más arriba - la cifra de más de 16.000 documentos recogidos.

${ }^{21}$ Los redactores del glosario aclaraban en las "Advertencias preliminares" de los fascículos $2(1962)$ a $8(1979)$ :
}

\begin{abstract}
5.- Cuando dentro de los límites cronológicos señalados (siglos IX-XI) disponemos de pocos ejemplos para ilustrar el significado de las palabras o cuando otras razones especiales así to aconsejan, recurrimos, caso de existir, a testimonios del siglo XII, pero no figuran aquellas palabras que en las fuentes catalanas se documentan por vez primera con posterioridad al año 1100.
\end{abstract}

En el fascículo 1, a-aragalius (1960), se mostraban menos estrictos, pues la advertencia número 5 acababa después de "siglo XII". En el fascículo 9, dotalis-dux (1985) no había advertencias. Por otra parte en los fasciculos 11, correspondiente a la letra $F(2001)$, y 12 , correspondiente a la letra G (2006), la redactora Teresa Gracia retoma el estilo del primer fascículo:

5. S'inclouen textos del segle XII quan es disposa de pocs exemples dins del límits cronològics assenyalats (s. IX-XI) o quan altres raons així ho aconsellen. 
encontraron ese documento y que no lo descartaron por tardío- o en la de los que tuvieran que encargarse de la segunda edición del glosario, unos y otros ante el conflicto personal de incluir o excluir conflictus.

Con respecto a la cuestión de la frecuencia de la aparición, hay que señalar que no es extraño que en el glosario queden recogidas palabras con un solo testimonio. En efecto, pongamos como ejemplos las siguientes voces pertenecientes al mismo fascículo 5 en el que el artículo conflictus, si se hubiera redactado, tendría que haberse publicado:

claustriger [ab claustrum] que lleva vida claustral; amante de la clausura: $\mathbf{s .}$ XI ex. Vita Petri Vrs. 13, p. 856: eductus ... a cella nouitiorum monachizatur ... fit mortuus uiuens inter uiuos ... fitque ... cilicinista $\ldots$ fit etiam inremotus claustriger impiger ${ }^{22}$.

De algunas palabras no se da definición, pues se trata de voces que ya existían en latín clásico y que sólo entran por la forma. En el siguiente caso, entrada sin definición, los redactores hallaron un sólo un ejemplo:

coaequalis escrito quoeq-: 973-995 LAntiq. I 46, f. 23, Mas 148 (Dei ... qui semper trinus in unitate manens quoeternus et quoequalis ante omnia secula $)^{23}$.

O incluso hay casos en que se da un solo testimonio que resulta, además, de significado dudoso:

coaequiperare $\left[a b\right.$ aequiperare] repartir equitativamente (?) ${ }^{1}: \mathbf{1 0 2 5}$ DipCarol., p. 446 (Dip. Berenguer Ramón I): si ... uobis instinctu diuino cogimus (pro-mur?) coequiperare commoda, ... nobis Domini gratiam fore credimus $^{24}$.

${ }^{22}$ Vita s. Petri Vrseoli (escrita, según parece, por un monje de Cuixà o de Ripoll), ed. J. MabILlON, Acta Sanctorum Ordinis Sancti Benedicti, VII (saec. V), Venetiae, 1737, pp. 851860.

${ }^{23}$ Libri Antiquitatum de la Catedral de Barcelona, Archivo de la Catedral de Barcelona; número de volumen, documento y folio. Se da a continuación la referencia del índice de J. MAS (Rúbrica dels «Libri Antiquitatum» de la Seu de Barcelona) en Notes històriques del bisbat de Barcelona, vols. IX-XII, Barcelona, 1909-1914.

Con respecto a la definición del término, $c f$ :

GAFFIOT: coaequalis, $e$ (cum, aeq-), 1 du même âge: PETR. 136, $1 \|$ coaequales, ium, m., f., personnes du même âge: in ludo inter coaequales discens JUST. 23, 4, 9, etant à l'école avec les enfants de son âge. $\| 2$ égal, pareil: VuLG. 2 Petr. $1,1$.

BLÁNQUEZ: coạequales, -ium, pl. de coaequalis, m. y f. pl. Personas de una misma edad, coetáneas. In ludo inter coaequales discens, Just., asistiendo a la escuela con los niños de su edad.

coaequalis, e. (de co por cum y aequalis). adj. Petr. De la misma edad, coetáneo, contemporáneo. \| Vulg. Parejo, igual. Véase coaequales.

${ }^{24}$ R. D’ABADAL I DE VINYALS, Els diplomes carolingis a Catalunya. Catalunya Carolíngia, vol. II/1-2, Barcelona, Institut d'Estudis Catalans, 1926-1950 y 1952. 


\begin{abstract}
${ }^{1}$ Significado conjetural; el preámbulo del diploma que citamos (carta otorgada por Berenguer Ramón I a los habitantes de Barcelona), de redacción obscura y en parte corrupto, es de muy dificil interpretación.
\end{abstract}

Pero leamos el texto en el que aparece la palabra conflictus. Se trata de un documento conservado en el Archivo de la Corona de Aragón, en el fondo de pergaminos de Ramón Berenguer III. Fechado en el año 1123, consiste en la declaración sacramental realizada por los testigos del testamento dictado por Udalardo, vizconde de $\operatorname{Bas}^{25}$. Dice así:

\begin{abstract}
Hec sunt conditiones sacramentorum ... de extrema voluntate cuiusdam hominis nomine Udalardi vicecomitis in conflicto militum apud Villam Mulorum mortui ... nos testes videlicet, Dalmacius Geraldi et frater eius Arnallus Geraldi, testificamus extremam voluntatem iam dicti Udalardi verbis ab eo nobis iniunctam etc. juramus super altare sancti Petri, quod situm est in ecclesia Sancti Privati de Bass, quia nos vidimus et udivimus et presentes ibi eramus et ab eo rogati extitimus quam iam dictus Udalardus volens ire versus Ispaniam in occursum scilicet ad Castrum de Curbins, sanus et incolumis in sua plena memoria et loquela ordinavit suam extremam voluntatem ... -Late condiciones XIIII Kalendas Januarii anno XVI Regni Ledovici Regis infra primos sex menses post obitum iamdicti testatoris. - S. Dalmacii Gerardi.- S. Arnalli Geraldi.- S. Guillelmus Raimundi capellanus Sancti Privati.- S. Guillelmus levita.S.Raimundi Arnalli de Beuda.- S. Bernaldi Eribaldi.- S.Arnalli Boni hominis sacerdotis.- S. Gaucefredi Guillelmi de Corneliano.
\end{abstract}

¿Cómo podemos traducir in conflicto militum ... mortui? Seguramente lo que nos salga más espontáneo sea "muerto en acción de guerra". ¿Y no estamos tomando conflictus como término concreto, con su significado primigenio, el de 'choque', el de 'operación', el de 'episodio de lucha en una guerra'? Es el sentido originario, el primer sentido del latín clásico y primer sentido, envejecido o desusual según las lenguas, en nuestras lenguas románicas. Pero, a pesar de ser un sentido clásico en latín, como decía F. Blatt en su preámbulo al primer fascículo - el de la letra L, publicado en 1957- del Novum Glossarium Mediae et Infimae Latinitatis, diccionario conocido entre los medievalistas como "Nuevo Du Cange", ¿no es necesario tener presente tanto lo que el latín medieval conserva como aquello en lo que se aleja del latín clásico para entender en qué consiste esa lengua bisagra, esa lengua que fluctúa como sus escribas y escritores, más o menos conscientes o inconscientes, según los momentos, de su alejamiento de la supuesta norma clásica, más o menos capaces de acercarse a ella o de inventársela? ${ }^{26}$

${ }^{25}$ ACA, Pergaminos de Ramon Berenguer III, $n .^{\circ}$ 256. Editado por F. MonsalvatJE Y FoSSAS, Noticias históricas, I-XXVI, Olot, 1899-1919, concretamente en el tomo XI (1901), pp. 416-417, y por Ll.G. CONSTANS I SERRATS, Diplomatari de Banyoles (822-1299), vol. I (1985), vol. II (1987), Banyoles, Centre d'Estudis Comarcals de Banyoles, 1985-1987, documento n $^{\circ}$ 129, vol. II, p. 120.

${ }^{26}$ Franz BLATT (dir.), Novum Glossarium Mediae Latinitatis ab anno DCCC usque ad annum $\operatorname{MCC},(\mathrm{L})$, Hafniae, Ejnar Munksgaard, 1957, "Avis au lecteur". 
Yo, puesta en el conflicto de elegir, decidiría incluir conflictus aunque fuera sólo atendiendo a su significado. Pero si eso no bastara, hay una razón más, contundente, para incluir el término en el glosario, una razón morfológica, que, en otras disciplinas no, pero en filología es una razón de peso. Efectivamente, en el sintagma in conflicto militum ... mortui observamos un cambio de declinación: el ablativo en "-o" no es propio de la cuarta declinación (conflictus, -us), cuyo ablativo es en "-u", sino de la segunda, declinación a la que pertenecería un supuesto *conflictus, $-i$.

\section{SIN PERSISTENCIA, SUBSTITUCIÓN}

Si una primera y rápida conclusión se puede extraer de la escasísima aparición del término conflictus en nuestros textos es que ese campo semántico tuvo que ser ocupado por otros substantivos. En efecto, uno de ellos, un germanismo, se impuso en toda la Romania y el dominio lingüístico correspondiente a nuestros documentos no fue una excepción: werra, guerra, tanto en un sentido restringido de 'lucha o querella violenta', como en el de 'guerra' en un sentido general. Paralelamente, el substantivo bellum, por antonomasia "la guerra" en el latín clásico, se especializó y acotó su significado para pasar a designar un 'conflicto o duelo judicial'; así nos aparece en los documentos latinos catalanes de la Alta Edad Media, en donde se emplean como sinónimos de bellum, con el mismo significado restringido, las nuevas acuñaciones batalia y duellum.

Todas estas palabras (guerra, bellum, batalia y duellum) están tratadas en los respectivos artículos del glosario ${ }^{27}$, al igual que ya desde que se compuso el fichero manual original se previó tratar e incluir en el glosario querela y querimonia, palabras también clásicas: querela (en principio 'queja', 'lamento' y de ahí 'queja', 'reclamación') y querimonia (también en principio 'queja', 'lamento' y de ahí 'disensión' y 'disentimiento') se circunscriben en los documentos leídos al campo léxico del lenguaje jurídico para aludir a la querella, al debate, al litigio producto de un conflicto de intereses. La misma previsión cabe suponer que se tuvo con otras dos palabras recogidas en el fichero manual y que aparecen a menudo acompañando a querimonia en contextos de conflicto jurídico: rancor (palabra clásica, pero entendida en un sentido que ya no corresponde al clásico de 'rencor') y rancura.

Y por último, ¿qué ocurre con el "conflicto interior", el vivido en una situación de tensión, sea provocada por una guerra, sea de otro origen? ¿No se plasma de ninguna manera en el latín altomedieval catalán? ¿Quizás con un romanismo, cuita, y con el empleo en un sentido desconocido por el latín

\footnotetext{
${ }^{27}$ Vid. guerra en el fascículo 12, letra G (2001); bellum y batalia en el fascículo 2, aragaliusbene (1962); de duellum, en el fascículo 9, dotalis-dux (1985), se remite a batalia, nota 1.
} 
clásico del término damnatio? ${ }^{28}$ Así parece que puede afirmarse, pero el "conflicto interior" se expresa sobre todo con una palabra también de impronta clásica: tribulatio, cuya inclusión en el glosario es, en estos momentos, cuando menos dudosa.

Efectivamente, la palabra, al ser clásica, no consta en el fichero manual. Sus elaboradores seguramente la encontraron en los textos que leyeron, pero la descartaron porque no ofrecía ningún matiz que la diferenciara de sus usos en latín clásico. Pero tribulatio, y su variante tribulacio, está atestiguada en la documentación digitalizada en 18 ocasiones. Todos los contextos evocan una situación de tensión personal, unos momentos más o menos dilatados de tribulación, de dificultad, de conflicto, no expresados de otro modo, sino con el substantivo en cuestión. Ahí está el concepto de "conflicto" que nos faltaba para completar su campo semántico, pero encarnado en una palabra que incumple las normas para su inclusión en un diccionario. Y está claro que es una norma de buen sentido la regla de no acumular, de no repetir lo que se puede evitar, lo que se puede encontrar en otros diccionarios, obras de referencia indiscutible.

El conflicto del lexicógrafo. Quizás esta intervención hubiera tenido que titularse así. O quizás, simplemente, el conflicto interior al que se alude en el título es el que afecta al redactor de un diccionario y la sensación de vértigo que puede invadirle al avistar las consecuencias de sus decisiones (consecuencias o secuelas, etimológicamente casi lo mismo).

Fecha de recepción del artículo: mayo 2008.

Fecha de aceptación y versión final: julio 2008.

${ }^{28}$ Vid. cuita en el fascículo 6, confrater-cyrographare (1971), donde se explica el origen del término romance como substantivo postverbal a partir del verbo catalán cuitar y su utilización como préstamo en el latín medieval catalán. Por otra parte, el significado especial de damnatio, cuyo uso en sentido figurado alude, según expresión de los redactores del GMLC, a una 'situación extraordinariamente angustiosa (como la de los condenados a las penas eternas)', a un ‘tormento', garantizó su aparición en el glosario; $c f$. fascículo 7, dalmatica-deouota (1976), s. v. 